\title{
Síndrome de Rapunzel. Una causa poco frecuente de obstrucción intestinal
}

\author{
Rapunzel Syndrome. A rare cause of intestinal obstruction
}

Dante A. Saldivar-Vera, Pedro A. Alvarado-Bahena*, Enrique Chávez-Serna, Jonathan Salgado-Vives y

Uraik F. Hernández-Bustos

Departamento de Cirugía General, Hospital General Regional 1 Querétaro, Instituto Mexicano del Seguro Social; División de Estudios de Posgrado, Universidad Autónoma de Querétaro. Querétaro, México

\section{Resumen}

Antecedentes: El síndrome de Rapunzel es la formación de un tricobezoar que se extiende más allá del intestino delgado. Desde su descubrimiento, pocos casos se han reportado en la literatura y con características clínicas variables, provocando complicaciones importantes como obstrucción intestinal. Actualmente, la laparotomía se considera el tratamiento de elección. Caso clínico: Paciente de sexo femenino que acude por presentar peritonitis de repetición asociada a catéter de diálisis peritoneal, anorexia, náuseas, vómito, sin canalizar ni presentar evacuaciones, tumoración en epigastrio, ansiedad, tricotilomanía y tricofagia. Se realiza el diagnóstico de síndrome de Rapunzel y se decide su ingreso a quirófano.

Palabras clave: Tricobezoar. Bezoar. Obstrucción. Tricotilomanía. Tricofagia.

\begin{abstract}
Background: Rapunzel syndrome is the formation of a trichobezoar that extends beyond the small intestine. Since its discovery few cases have been reported in the literature with variable clinical characteristics, causing important complications such as intestinal obstruction. Laparotomy is currently considered the treatment of choice. Case report: We present the clinical case of a patient who presented with recurrent peritonitis associated with a peritoneal dialysis catheter, anorexia, nausea, vomiting, without channeling or presenting evacuations, epigastric tumor, anxiety, trichotillomania and trichophagia. Rapunzel syndrome is diagnosed and admission to the operating room is decided.
\end{abstract}

Key words: Trichobezoar. Bezoar. Obstruction. Trichotillomania. Trichophagia.

\section{Introducción}

La tricotilomanía es una condición de deseo irresistible de desprenderse el propio cabello¹. La tricofagia, en cambio, es comerse el cabello ${ }^{2}$, propiciando la formación de tricobezoares, que suelen residir en el estómago ${ }^{3}$ y rara vez pueden extenderse al intestino delgado $^{4}$, donde se denomina síndrome de Rapun$z^{5}{ }^{5}$. Este fue originalmente descrito por Vaughan en 
1968. Desde entonces, pocos casos se han reportado en la literatura y con características clínicas variables. Se han usado distintos criterios por diferentes autores para reportar sus casos de síndrome de Rapunzel. Algunos lo han definido como un tricobezoar gástrico con una cola que se extiende hasta la válvula ileocecal; otros como simplemente un tricobezoar con una cola larga, que puede extenderse al yeyuno o al íleon, e incluso hay quienes lo definen como un tricobezoar de cualquier tamaño que se presenta con una obstrucción intestinal ${ }^{6,7}$. El $90 \%$ de los casos son mujeres y el $80 \%$ son menores de 30 años ${ }^{8}$. Pueden permanecer sin diagnosticar durante años, condicionando complicaciones graves 9 .

\section{Caso clínico}

Mujer de 22 años, con antecedente de enfermedad renal crónica en estadio $V$ en tratamiento con diálisis peritoneal, hipertensión arterial, tricotilomanía y tricofagia desde los 4 años y ansiedad generalizada sin apego al tratamiento. Acude al servicio de urgencias por presentar cuadros repetitivos de peritonitis asociada a catéter de diálisis peritoneal en tratamiento con antibiótico de amplio espectro, sin mejoría aparente, además de presentar náuseas, vómitos, intolerancia a la vía oral y dolor epigástrico. Refiriere no canalizar gases y no presentar evacuaciones desde hace 2 días, motivo por el cual se decide su ingreso hospitalario y se solicitan radiografía abdominal (Fig. 1) y tomografía computarizada toracoabdominal (Fig. 2), en la que se aprecia una imagen heterogénea de localización intraluminal en el estómago, la cual abarca la totalidad de la cámara gástrica con extensión hacia la primera porción del duodeno, aumento de la densidad y desplazamiento de las asas intestinales. Se decide su intervención quirúrgica realizando laparotomía exploradora, en la que se encuentra gastromegalia. Se procede a realizar gastrotomía en la cara anterior, observando un tricobezoar (Figs. 3 y 4 ) de $52 \mathrm{~cm} \times 11 \mathrm{~cm}$, con un peso de $1900 \mathrm{~kg}$, ocupando la luz gástrica en su totalidad con extensión hacia la primera porción del duodeno. Se extrae y se procede al cierre del estómago, y posteriormente a revisión de la cavidad abdominal en búsqueda de perforaciones o lesiones intestinales, con retiro de catéter Tenckhoff. La paciente pasa de recuperación a piso de nefrología, con adecuada evolución posquirúrgica. Se inicia terapia de sustitución renal mediante hemodiálisis y se interconsulta al servicio de psiquiatría para manejo de patologías asociadas.

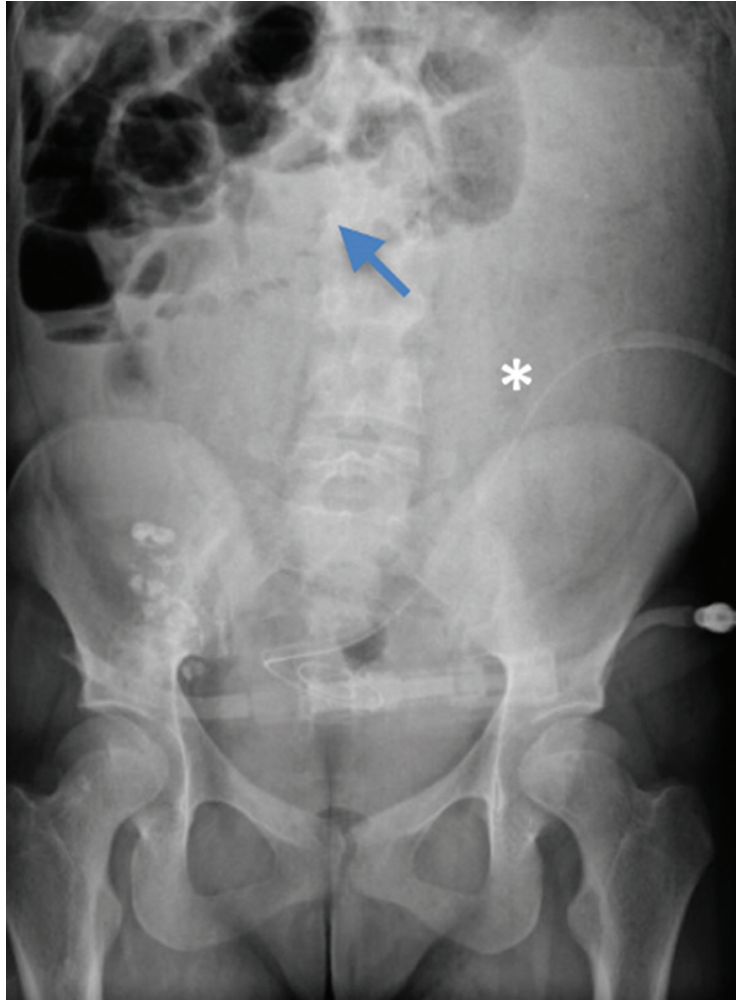

Figura 1. Radiografía anteroposterior de abdomen, en la que se observa la presencia de un catéter de Tenckhoff localizado en el hueco pélvico, así como un aumento de densidad que dibuja la cámara gástrica aumentada de tamaño (asterisco), la cual condiciona el desplazamiento de las asas intestinales distendidas hacia el hipocondrio derecho (flecha).
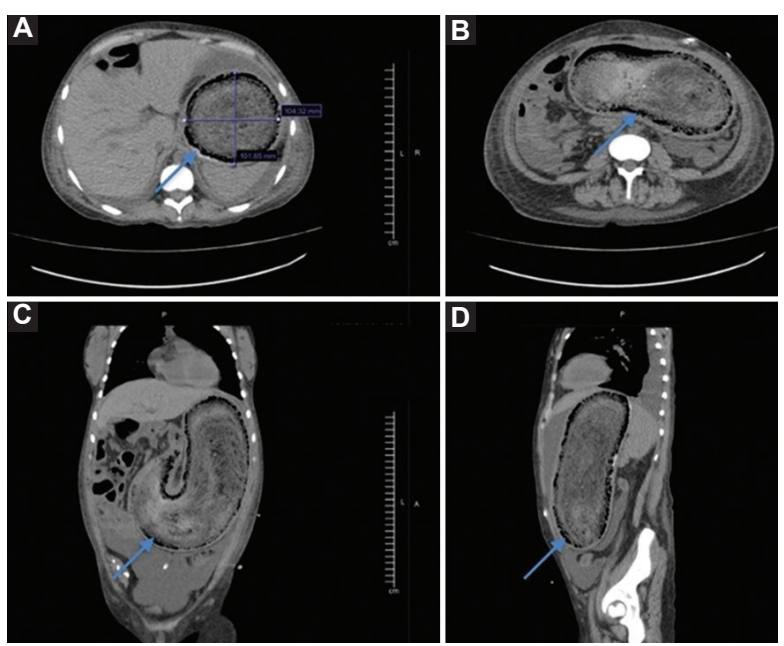

Figura 2. A y B: estudio tomográfico simple de abdomen en corte axial. C: corte coronal. D: corte sagital. Se aprecia una imagen heterogénea de localización intraluminal en el estómago (flecha), con burbujas de aire en su superficie, la cual abarca la totalidad de la cámara gástrica y se extiende hacia la primera porción del duodeno, con unas dimensiones máximas de $23 \times 10 \times 10 \mathrm{~cm}$. El contenido abdominal está desplazado hacia el hipocondrio derecho. 


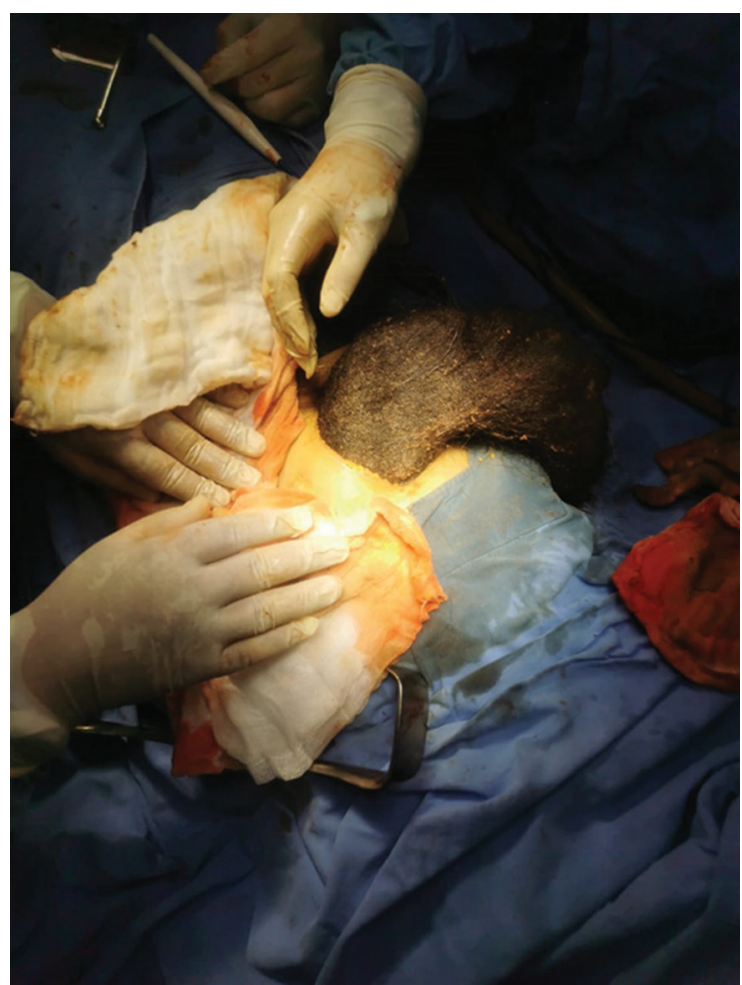

Figura 3. Pieza quirúrgica obtenida de tricobezoar, con unas dimensiones de $52 \times 11 \mathrm{~cm}$ y un peso de $1900 \mathrm{~g}$.

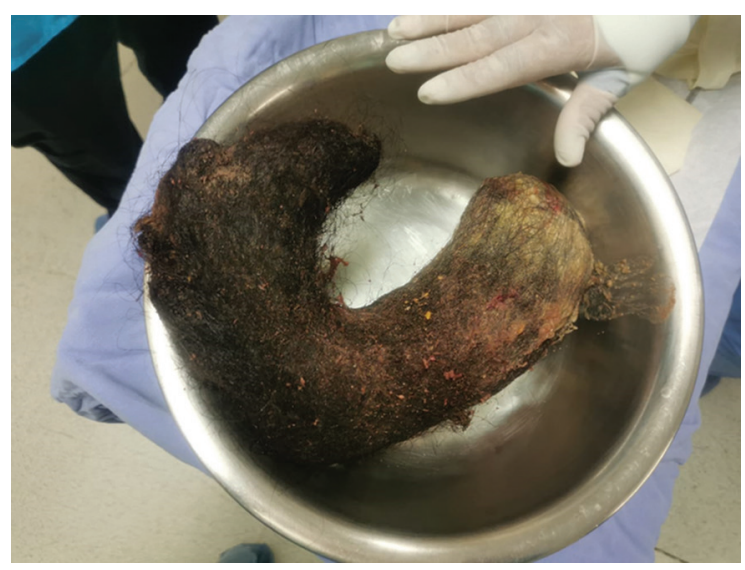

Figura 4. Pieza quirúrgica de tricobezoar.

\section{Discusión}

El síndrome de Rapunzel generalmente ocurre en mujeres con afecciones psiquiátricas ${ }^{10}$. Es causado por una afección al desprenderse el propio cabello (tricotilomanía) y luego comérselo (tricofagia) ${ }^{11}$. Estos padecimientos pueden asociarse con otros trastornos psiquiátricos, como ansiedad generalizada, pica, trastorno obsesivo-compulsivo, depresión mayor y anorexia nerviosa ${ }^{12}$. La condición ocurre debido a la falla de la peristalsis gástrica para eliminar el cabello del estómago, con su eventual conglomerado. Permanece asintomática o puede presentar dolor abdominal, náuseas, vómitos, saciedad y pérdida de peso $^{12}$. En la exploración física se observa una masa abdominal bien definida, lisa, firme y móvil en la región epigástrica $^{13}$. Puede causar muchas complicaciones, como obstrucción de la salida gástrica (26\%), hemorragia gastrointestinal $(10 \%)$, ictericia obstructiva, pancreatitis aguda, obstrucción del intestino delgado, perforación, peritonitis e invaginación intestinal ${ }^{13-15}$. Los métodos radiológicos, como la radiografía abdominal y la tomografía computarizada, y la ecografía muestran estructuras calcificadas, granulares o en forma de remolino de material sólido y gaseoso, o defectos de llenado dentro del estómago. El diagnóstico se confirma mediante tomografía computarizada con contraste oral ${ }^{16,17}$. Respecto al tratamiento para eliminar un tricobezoar, no se encuentra estandarizado e incluye disolución química, remoción endoscópica, procinéticos adyuvantes y cirugía. En una revisión se demostró una tasa de éxito del $75 \%$ para el tratamiento laparoscópico y del $99 \%$ con la cirugía abierta ${ }^{18,19}$. Aunque el éxito es mayor con la laparotomía, existe una mayor probabilidad de complicaciones. Es importante que el cirujano que se enfrenta a estos casos tenga en cuenta el estado clínico del paciente y la gravedad de la obstrucción para elegir el tipo de procedimiento. En un paciente que solo tiene un bezoar con obstrucción parcial o que está confinado solo al estómago, el beneficio de la laparoscopia es mayor.

\section{Conclusiones}

El síndrome de Rapunzel está causado por dos afecciones importantes, la tricotilomanía y la tricofagia, sobre todo en mujeres jóvenes con trastornos psiquiátricos. Los síntomas comunes son dolor abdominal, náuseas, vómitos y pérdida de peso. Puede complicarse con obstrucción de la salida gástrica, ictericia obstructiva, hemorragia gastrointestinal, pancreatitis aguda, obstrucción del intestino delgado, perforación, peritonitis e invaginación intestinal. Actualmente no hay un régimen estandarizado para el tratamiento; sin embargo, la laparoscopia y la laparotomía son los métodos de elección, con una mayor tasa de éxito con esta última. No obstante, hay que individualizar siempre según el grado de obstrucción y el tamaño del tricobezoar, además de la comorbilidad de los pacientes. 


\section{Responsabilidades éticas}

Protección de personas y animales. Los autores declaran que para esta investigación no se han realizado experimentos en seres humanos ni en animales.

Confidencialidad de los datos. Los autores declaran que han seguido los protocolos de su centro de trabajo sobre la publicación de datos de pacientes.

Derecho a la privacidad y consentimiento informado. Los autores han obtenido el consentimiento informado de los pacientes y/o sujetos referidos en el artículo. Este documento obra en poder del autor de correspondencia.

\section{Financiamiento}

Los autores declaran que no existe financiamiento.

\section{Conflicto de intereses}

Los autores declaran no tener conflicto de intereses.

\section{Bibliografía}

1. Morales-Fuentes B, Camacho-Maya U, Coll-Clemente FL, Vázquez-Minero JC. Trichotillomania, recurrent trichobezoar and Rapunzel syndrome: case report and literature review. Cir Cir. 2010;78:265-6.
2. Álvarez J, Álvarez E, Clint J, Sauret J. Hallazgos radiológicos en el síndrome de Rapunzel (tricobezoar). SEMERGEN. 2009;35:350 2.

3. Latorre Díez A, González Soler JJ, Suárez Rodríguez B. Weight loss and abdominal pain in a young woman: gastric trichobezoar. Med Clin (Barc). 2018;150:e33.

4. Varma A, Sudhindra BK. Trichobezoar with small bowel obstruction. Indian J Pediatr. 2008;65:761-3.

5. Vila S, García C, Piscoya A, de los Ríos R, Pinto JL, Huerta-Mercado J, et al. Giant gastroduodenal trichobezoar: Rapunzel syndrome. Am J Gastroenterol. 2009;104:2864-5.

6. Naik S, Gupta V, Naik S, Rangole A, Chaudhary AK, Jain P, et al. Rapunzel syndrome reviewed and redefined. Dig Surg. 2007;24:157-61.

7. Beristain-Silva JL, Cordero-Barberena R, Beristain-Hernández JL. Rapunzel syndrome: a rare cause of abdominal pain. Rev Gastroenterol Mex. 2016;81:178-9.

8. Bargas Ochoa M, Xacur Hernández M, Espadas Torres M, Quintana Gamboa A, Tappan Lavadores I, Méndez Domínguez N. Rapunzel syndrome with double simultaneous trichobezoar in a teenager: clinical case report. Rev Chil Pediatr. 2018;89:98-102.

9. Mirza MB, Talat N, Saleem M. Gastrointestinal trichobezoar: an experience with 17 cases. J Pediatr Surg. 2020;55:2504-9.

10. Grimm Brothers. Rapunzel. Translated by Godwin-Jones R. Richmond: Virginia Commonwealth University Department of Foreign Languages; 1994-1999.

11. Gonuguntla V, Joshi DD. Rapunzel syndrome: a comprehensive review of an unusual case of trichobezoar. Clin Med Res. 2009;7:99-102.

12. Gorter RR, Kneepkens CM, Mattens EC, Aronson DC, Heij HA. Management of trichobezoar: case report and literature review. Pediatr Surg Int. 2010;26:457-63.

13. Ersoy YE, Ayan F, Ayan F, Ersan Y. Gastro-intestinal bezoars: thirty-five years experience. Acta Chir Belg. 2009;109:198-203.

14. Ullah W, Saleem K, Ahmad E, Answer F. Rapunzel syndrome: a rare cause of hypoproteinaemia and review of literature. BMJ Case Rep. 2016;2016:bcr2016216600.

15. Williams RS. The fascinating history of bezoars. Med J Aust. 1986;145:613-4.

16. Couceiro A, Viveiro C, Capelão G, Nobre J, Laureano M, Gonçalves I, et al. Trichobezoar - a rare cause of abdominal mass and gastric outlet obstruction. GE Port J Gastroenterol. 2016;23:50-3.

17. Phillips MR, Zaheer S, Drugas GT. Gastric trichobezoar: case report and literature review. Mayo Clin Proc. 1998;73:653-6.

18. Benatta MA. Endoscopic retrieval of gastric trichobezoar after fragmentation with electrocautery using polypectomy snare and argon plasma coagulation in a pediatric patient. Gastroenterol Rep (Oxf). 2016;4:251-3.

19. Tudor ECG, Clark MC. Laparoscopic-assisted removal of gastric trichobezoar; a novel technique to reduce operative complications and time. J Pediatr Surg. 2013;48:e13-5. 